\title{
Chromosome and genomic relationships between the diploid species Avena strigosa, A. eriantha and the tetraploid A. maroccana
}

\author{
J. M. LEGGETT* \\ Institute of Grassland and Environmental Research, Plas Gogerddan, Aberystwyth, Ceredigion SY23 3EB, U.K.
}

\begin{abstract}
Chromosome pairing in the diploid species Avena strigosa (genome AsAs), A. eriantha (genome $\mathrm{CpCp}$ ) and the hybrid between them was broadly similar to a previous report. The derived autoallotetraploid $(A \text {. strigosa } \times A \text {. eriantha })^{2}$ showed greater variation of chromosome pairing than expected, particularly with regard to the frequency of rod bivalents and univalents. The hybrid $(A \text {. strigosa } \times A \text {. eriantha })^{2} \times A$. maroccana (genomes AACC) would be expected to form 14 bivalents if the AsAs and CpCp genomes of $A$. strigosa and $A$. eriantha were completely homologous, respectively, to the AA and $\mathrm{CC}$ genomes of $A$. maroccana. Chromosome pairing in the hybrid was poor, however, indicating that it is unlikely that $A$. eriantha or $A$. strigosa participated in the formation of the tetraploid $A$. maroccana.
\end{abstract}

Keywords: Avena, chromosome pairing, interspecific hybrids, phylogeny.

\section{Introduction}

The wild tetraploid oat Avena maroccana Gdgr. $(2 n=4 \mathrm{x}=28$; genomes AACC) is thought to be the evolutionary link between the diploids and the cultivated hexaploid oat $A$. sativa L. $(2 n=6 \mathrm{x}=42$; genomes AACCDD). The $\mathrm{A}$ and $\mathrm{C}$ genomes occur at all three ploidy levels, whereas the $\mathrm{D}$ genome is found only in the hexaploid species (Leggett \& Markhand, 1995).

This paper describes the meiotic chromosome pairing of $A$. strigosa Schreb. (genome AsAs) and $A$. eriantha Dur. (genome $\mathrm{CpCp}$ ), the hybrid between them, the derived allotetraploid of this cross ( $A$. strigosa $\times A$. eriantha $)^{2}$ and the hybrid produced by crossing this allotetraploid with $A$. maroccana. The results are discussed in relation to the evolution of A. maroccana.

\section{Materials and methods}

In the diploid cross, $A$. strigosa cultivar Saia was the female parent, and the accession $\mathrm{Cc} 7056$ of $A$. eriantha, originally collected in Iraq, was the male parent. The autoallotetraploid was derived by treating tillers of the $A$. strigosa $\times A$. eriantha hybrid with colchicine.

\footnotetext{
*Correspondence. E-mail: mike.leggett@bbsrc.ac.uk
}

The contrived allotetraploid was the female parent in the cross with $A$. maroccana accession Cc7071, originally collected in Morocco. Embryo rescue, plant growth and preparation of plant material for microscopic examination was as described by Leggett (1992).

\section{Results}

The meiotic chromosome pairing of $A$. eriantha and $A$. strigosa was regular, with the formation of seven bivalents (Table 1), although the number of ring bivalents was 5 per cent lower in $A$. strigosa than in $A$. eriantha. In the hybrids $A$. strigosa $\times A$. eriantha (Av5569/1 and /2) no pollen mother cells (PMCs) were recorded with fewer than four univalents and synapsis completely failed in 17.69 per cent of PMCs. Fewer than four bivalents (and no multivalents) were recorded in 76.18 per cent of PMCs and quadrivalents and trivalents were observed in only 2.12 per cent of PMCs. The maximum chromosome pairing was five bivalents and four univalents per PMC and the minimum, 14 univalents (Table 1).

The colchicine-treated A. strigosa $\times A$. eriantha plant (Av5569/2) ${ }^{2}$ contained only 27 chromosomes. Thirteen bivalents and a univalent (the monosome) were recorded in 45.71 per cent of PMCs, the 
Table 1 Chromosome pairing at meiotic metaphase I of Avena eriantha, A. strigosa, the hybrid $A$. strigosa $\times$ A. eriantha, the derived autoallotetraploid $(A \text {. strigosa } \times A \text {. eriantha })^{2}$, and the hybrid $(A \text {. strigosa } \times A \text {. eriantha })^{2} \times A$. maroccana

\begin{tabular}{lcccccccc}
\hline & & & \multicolumn{6}{c}{ Mean chromosome pairing and chiasmata frequency per pollen mother } \\
(range in parentheses) cell
\end{tabular}

*A. strigosa $\times A$. eriantha.

$\dagger(\text { A. strigosa } \times \text { A. eriantha })^{2}$, i.e. Av 5569 chromosome doubled.

$\ddagger(\text { A. strigosa } \times A \text {. eriantha })^{2} \times A$. maroccana.

remaining PMCs contained from nine to 12 bivalents and three to nine univalents (Table 1). No PMCs containing multivalents were observed.

Chromosome pairing in the hybrids Av5616/2 and Av5616/3 between $A$. maroccana and the chromosome doubled hybrid (Av5569) ${ }^{2}$ (Table 1) was highly irregular, all PMCs containing univalents, and 77.34 per cent containing multivalents. The maximum chromosome pairing recorded was 1IV 2III 6II and 5I, and the minimum was 4II and 19I.

\section{Discussion}

Only one other hybrid between AA and CC genome diploid oats has been reported previously (Nishiyama \& Yabuno, 1975), which also involved the species $A$. eriantha and $A$. strigosa. Nishiyama \& Yabuno (1975) observed just over 50 per cent of PMCs with no chromosomes paired and no multivalents were recorded in any PMC, whereas in the present study complete failure of synapsis was recorded in only 17.7 per cent of PMCs and a low frequency of multivalent formation caused by an intragenomic translocation was noted.

The low mean number of bivalent associations (1.45), all rod configurations, together with the high number of unpaired chromosomes, indicates that there is little homology/homoeology between the AsAs and CpCp genome chromosomes of these two diploid species. This lack of homology is borne out by the in situ hybridization studies of Leggett \& Markhand (1995), who demonstrated that the AA and $\mathrm{CC}$ genomes are quite distinct at the DNA level.

Chromosome pairing in the synthetic allotetraploid derived from the doubling of the chromosome complement of the $A$. strigosa $\times A$. eriantha hybrid (Table 1) would be expected to have complete and regular bivalent pairing, as each chromosome would have its true homologue with which to pair. However, the 'chromosome-doubled' plant $(\text { Av5569) })^{2}$ contained only 27 chromosomes and one would therefore expect a univalent in every PMC. The mean number of univalents per PMC (2.66) was higher than expected, even taking the monosomic into account. The fact that 34 per cent of PMCs contained three univalents suggests that one pair of chromosomes is frequently failing to pair, in addition to the monosome. However, there is no evidence to suggest that the same chromosome pair is involved in each cell.

Chromosome pairing in the hybrids Av5616/2 and Av5616/3 would be expected to form 14 bivalents, if the AsAs and CpCp genomes of $A$. strigosa and $A$. eriantha were completely homologous to the AA and $\mathrm{CC}$ genomes of $A$. maroccana, respectively. Meiotic chromosome pairing of the hybrids (both of which contained only 27 chromosomes), however, was

(C) The Genetical Society of Great Britain, Heredity, 80, 361-363. 
poor, with a mean of 11 chromosomes remaining as univalents (Table 1).

Multivalents were recorded in 77 per cent of PMCs, a frequency over twice as high as the combined multivalent frequency reported in the triploid hybrids $A$. strigosa $\times A$. maroccana (Sadanaga et al., 1968) and $A$. eriantha $\times A$. maroccana (Kummer $\&$ Miksh, 1977). Interpreting these differences is difficult because the genotypes used to produce the triploid hybrids differed from those used in this study, which could, in part, account for the discrepancies.

Multivalent formation in the Av5616 hybrids could result from homologous/homoeologous chromosome pairing, or from translocations, the latter especially if the diploidizing mechanism as described by Rajhathy \& Thomas (1974) is operative in this hybrid. The general lack of homology between the AsAs and $\mathrm{CpCp}$ genomes in the diploid hybrids reported here and by Nishiyama \& Yabuno (1975), together with the chromosome pairing data of the triploid hybrids involving AA and $\mathrm{CC}$ genome diploid species and A. maroccana (Sadanaga et al., 1968; Kummer \& Miksh, 1977) and the synthetic allotetraploid Av5616, indicate that it is probable that the multivalent formation recorded in the Av5616 hybrids results from translocations between $\mathrm{AA}$ and CC genome chromosomes.

In situ hybridization studies by Leggett et al. (1994) have shown that in A. maroccana there are indeed four CC genome segments translocated onto AA genome chromosomes (at least three of which appear to be of a nonreciprocal nature) and a further AA genome segment translocated onto a CC genome chromosome. Nevertheless, chromosome pairing in $A$. maroccana is confined to bivalent formation. This lends further weight to the theory that the multivalent formation in the Av5616 hybrid described above is more likely to be caused by translocations than by homoeologous chromosome pairing.
In conclusion, it is evident from the in situ hybridization studies of Leggett et al. (1994) that $A$. maroccana contains both AA and CC genomes and the data presented here indicate that the $\mathrm{CpCp}$ genome of $A$. eriantha, and the AsAs genome of $A$. strigosa, are only distantly related to the $\mathrm{AA}$ and $\mathrm{CC}$ genomes of $A$. maroccana at the chromosome level and are, therefore, unlikely to have played any role in the formation of this tetraploid.

\section{Acknow ledgements}

Financial support from the BBSRC (UK) during the execution of these studies is gratefully acknowledged by the author.

\section{References}

KUMMER, M. AND MIKSH, G. 1977. Successful hybridization between species Avena pilosa M. Bieb. \& Avena magna Murphy et Terrel. Cereal Res. Comm., 5, 249-254.

LEGGETT, J. M. 1992. Further hybrids involving the perennial autotetraploid oat Avena macrostachya. Genome, 35, 273-275.

LEGGETT, J. M. AND MARKHAND, s. M. 1995. The genomic identification of some monosomics of Avena sativa $\mathrm{L}$. cv. Sun II using GISH. Genome, 38, 747-751.

LEGGETt, J. M., THOMAS, H. M., MEREDITH, M. R., HUMPHREYS, M. W., MORGAN, W. G., THOMAS, H. AND KING, I. P. 1994. Intergenomic translocations and the genomic composition of Avena maroccana Gdgr. revealed by FISH. Chromosome Res., 2, 163-164.

NISHIYAMA, I. AND YABUNO, T. 1975. Meiotic chromosome pairing in two interspecific hybrids and a criticism of the evolutionary relationship of diploid Avena. Jap. J. Genet., 50, 443-451.

Rajhathy, T. AND thomas, H. 1974. Cytogenetics of oats (Avena L.). Miscellaneous Publ. Genet. Soc. Canada. No.2. Ottawa, Ontario, Canada.

SADANAGA, K., ZILLINSKY, F. J., MURPHY, H. C. AND SMITH, R. T. 1968. Chromosomes associations in triploid, tetraploid and pentaploid hybrids of Avena magna $(2 \mathrm{n}=28)$. Crop Sci., 8, 594-597. 